\title{
Spreading Addictions Care Across Oregon's Rural and Community Hospitals: Mixed-Methods Evaluation of an Interprofessional Telementoring ECHO Program
}

\author{
Honora Englander, $M D^{1,2}$ (D) Alisa Patten, $\mathrm{MA}^{2}$, Rachel Lockard, $\mathrm{MPH}^{3}$, \\ Matthew Muller, $B A^{4}$, and Jessica Gregg, $M D, P h D^{2,5}$
}

\begin{abstract}
'Division of Hospital Medicine, Department of Medicine, Oregon Health \& Science University, Portland, OR, USA; '2Section of Addiction Medicine, Department of Medicine, Oregon Health \& Science University, Portland, OR, USA; ${ }^{3}$ School of Medicine, Oregon Health \& Science University, Portland, OR, USA; ${ }^{4}$ Knight Cardiovascular Institute, Oregon Health \& Science University, Portland, OR, USA; ${ }^{5}$ Division of General Internal Medicine, Department of Medicine, Oregon Health \& Science University, Portland, OR, USA.
\end{abstract}

\begin{abstract}
BACKGROUND: Despite evidence of effectiveness, most US hospitals do not deliver hospital-based addictions care. ECHO (Extension for Community Healthcare Outcomes) is a telementoring model for providers across diverse geographic areas. We developed and implemented a substance use disorder (SUD) in hospital care ECHO to support statewide dissemination of best practices in hospital-based addictions care.
\end{abstract}

OBJECTIVES: Assess the feasibility, acceptability, and effects of ECHO and explore lessons learned and implications for the spread of hospital-based addictions care.

DESIGN: Mixed-methods study with a pre-/post-intervention design.

PARTICIPANTS: Interprofessional hospital providers and administrators across Oregon.

INTERVENTION: A 10-12-week ECHO that included participant case presentations and brief didactics delivered by an interprofessional faculty, including peers with lived experience in recovery.

APPROACH: To assess feasibility and acceptability, we collected enrollment, attendance, and participant feedback data. To evaluate ECHO effects, we used pre-/postECHO assessments and performed a thematic analysis of open-ended survey responses and participant focus groups.

KEY RESULTS: We recruited 143 registrants to three cohorts between January and September 2019, drawing from 32 of Oregon's 62 hospitals and one southwest Washington hospital. Ninety-six (67.1\%) attended at least half of ECHO sessions. Participants were highly satisfied with ECHO. After ECHO, participants were more prepared to treat SUD; however, prescribing did not change. Participants identified substantial gains in knowledge and skills, particularly regarding the use of medications for opioid use disorder; patient-centered communication

An earlier version of this work was presented at the Association for Multidisciplinary Education and Research in Substance use and Addiction (AMERSA) National Conference in Boston, MA on November 9, 2019.

Electronic supplementary material The online version of this article (https://doi.org/10.1007/s11606-020-06175-5) contains supplementary material, which is available to authorized users.

Received April 24, 2020

Accepted August 20, 2020

Published online September 3, 2020 with people who use drugs; and understanding harm reduction as a valid treatment approach. ECHO built a community of practice and reduced provider isolation. Participants recognized the need for supportive hospital leadership, policies, and SUD resources to fully implement and adopt hospital-based SUD care.

CONCLUSIONS: A statewide, interprofessional SUD hospital care ECHO was feasible and acceptable. Findings may be useful to health systems, states, and regions looking to expand hospital-based addictions care.

KEY WORDS: substance-related disorders; hospitalization; substance use treatment; ECHO model; continuing medical education; case-based learning.

$\mathrm{J}$ Gen Intern Med 36(1):100-7

DOI: $10.1007 / \mathrm{s} 11606-020-06175-5$

(C) Society of General Internal Medicine 2020

\section{INTRODUCTION}

The addiction epidemic is hitting hospitals hard. People with substance use disorder (SUD) have high rates of hospitalization, readmission, and death. ${ }^{1,2}$ Most people with SUD who present to general medical hospitals are not seeking addictions treatment, but hospitalization can be a pivotal moment to engage patients and initiate SUD care. ${ }^{3,4}$ Hospital-based addictions care is associated with improved patient experience, ${ }^{5,6}$ improved post-hospital SUD treatment engagement, ${ }^{7,8}$ decreased substance use severity, ${ }^{7,9}$ improved provider experience, ${ }^{10}$ and lower costs. ${ }^{7}$ Yet, most hospitals do not treat addiction. While increasingly, academic medical centers are implementing dedicated addiction consult services, ${ }^{11-13}$ little is known about how to spread best practices and deliver hospital-based addictions care across rural and community hospitals, which have different resources, cultures, opportunities, and challenges than academic centers.

Project ECHO (Extension for Community Healthcare Outcomes) is a distance education model that connects specialists with remote providers via a simultaneous video link. ${ }^{14} \mathrm{ECHO}$ is founded on the principle of de-monopolizing medical knowledge, where specialists share expertise and provide 
telementorship and guided practice to community providers to deliver high-quality specialized care to patients in their own communities. ${ }^{14}$ Our hospital is an urban academic medical center that offers regional ECHO programming ${ }^{15}$ and has a well-established interprofessional inpatient addiction consult service, called the Improving Addictions Care Team (IMPACT). ${ }^{3,16}$ We developed and piloted a SUD in hospital care ECHO to spread best practices in hospital-based addictions care across Oregon. To our knowledge, this is the first evaluation of a regional or statewide effort to support broad-scale adoption of hospital-based SUD care.

This study (1) describes the feasibility and acceptability of a SUD in hospital care ECHO (herein called ECHO), (2) evaluates $\mathrm{ECHO}$ effects on interdisciplinary providers' knowledge and practices regarding SUD treatment, and (3) describes lessons learned and implications for spread of hospital-based addictions care across community hospitals.

\section{METHODS}

\section{Setting}

Oregon is a large rural state with 62 hospitals, including 25 urban, 30 small rural, and 7 frontier hospitals. Small rural hospitals have 50 or fewer beds and are located at least 30 miles from an acute care hospital. Frontier hospitals are located in counties with six or fewer people per square mile. To our knowledge, in Oregon at the outset of this ECHO, two hospitals offered hospital-based addiction consultation.

\section{ECHO Development and Description}

ECHO includes participant case presentations followed by brief didactics. ${ }^{17}$ We developed the didactic curriculum based on our hospital's existing SUD in ambulatory care ECHO, our experience developing IMPACT implementation tools, ${ }^{16}$ and input from hospital teams from urban, rural, and frontier hospitals and the Oregon Association of Hospitals and Health Systems (OAHHS).

IMPACT experience and stakeholder feedback highlighted the importance of interprofessional training and collaboration. Thus, we recruited an interprofessional ECHO faculty, including IMPACT physicians, social worker, and peer mentor, plus a hospital pharmacist and nurse. Faculty developed a 10-week curriculum and detailed learning objectives. We planned to adapt ECHO to incorporate participant feedback. After cohort 1 , we added two additional sessions. While stakeholders identified a need for SUD training, many noted that competing clinical demands for frontline staff and hospitalists' typical "week-on, week-off" schedules might threaten feasibility. To address this, we made sessions as high yield as possible, hoping to promote strong attendance.

ECHO included weekly hour-long sessions (12:001:00 p.m., Wednesdays), including a participant case presentation followed by a brief lecture. Before presenting, participants completed a case form (ESM Appendix). Lecture topics broadly addressed hospital addictions care, including medication for opioid use disorder, harm reduction, managing active substance use in hospital, and institutional buy-in for systems change (Table 1). We supplemented lectures with online tools, resources, and published literature, and we distributed case recommendations after each session. An online repository includes links to lectures and resources. ${ }^{18}$ Faculty was available outside of ECHO to consult on difficult cases or systems change.

ECHO was funded through the Oregon State Targeted Response Grant ${ }^{19}$ and was free for all participants.

\section{ECHO Evaluation}

We conducted a mixed-methods pilot study, ${ }^{20}$ with a pre- $/$ post-intervention design, to assess the feasibility and acceptability of ECHO and to explore its effectiveness. We collected quantitative survey data and qualitative short-answer surveys and focus group interviews, using qualitative methods to explain and elaborate quantitative findings. We developed surveys (ESM Appendix) based on literature review and expert input and adapted an existing preparedness scale. ${ }^{21} \mathrm{We}$ elicited feedback through participant focus groups (ESM Appendix) after each cohort. The OHSU Institutional Review Board determined that this study was not human subjects research.

Recruitment and Eligibility. We recruited participants through targeted email communication via the Oregon ECHO Network and OAHHS. For cohort 1, we delivered an OAHHS-sponsored webcast and outreached to hospitals with high rates of SUD-related admissions. We encouraged participants to invite colleagues to future cohorts and allowed participants to enroll multiple times. We offered free continuing education and maintenance of certification credits for eligible participants. $^{22}$

Eligible participants worked in an Oregon or Southwest Washington hospital, or a partnering organization (e.g.,

\section{Table 1 ECHO Curriculum}

\begin{tabular}{ll}
\hline \hline Session & Title (faculty lecturer discipline) \\
\hline 1 & Introduction to ECHO and SUD 101 (MD) \\
2 & Buprenorphine in Hospital Care (MD) \\
3 & Methadone in Hospital Care (MD) \\
4 & Trauma-Informed Care in a Hospital Setting (social worker) \\
5 & Faculty Role Play I: Acute Opioid Withdrawal, Diagnosing \\
6 & SUD, Discussing Safer Use (RN, MD, peer, social worker) \\
7 & Integrating Peers in Hospital Care (peer, MD) \\
8 & Overdose Prevention and Harm Reduction (pharmacist, peer) \\
9 & Methamphetamine Use Disorder (MD) \\
10 & Community SUD Treatment Settings (social worker) \\
11 & Acute Pain in the Setting of Opioid Use Disorder (MD, pain \\
12 & Frovider) \\
Extra & peer, social worker) \\
\hline
\end{tabular}

ECHO materials are available in an online repository that includes lecture slides and a summary with links to supplementary training materials and point-of-care resources ${ }^{18}$ 
accountable care organization, community addiction organization). We recruited interprofessional administrators and providers, including hospitalists, nurse practitioners, physician assistants, nurses, social workers, pharmacists, and case managers.

Data Collection. We collected data between January 2019 and January 2020. Participants completed a baseline survey between weeks 0 and 3 and a post-ECHO survey after ECHO completion. Surveys asked about SUD knowledge, preparedness, and practice; and open-ended questions about barriers and facilitators to implementing SUD care. Participants completed weekly post-session evaluations in an online database. We invited all participants to a semistructured focus group after ECHO.

Feasibility and Acceptability. We collected information on participant recruitment and attendance. We measured acceptability from satisfaction scores from per-session evaluations, post-ECHO surveys, and focus groups.

Intervention Effectiveness. We compared pre- and postintervention measures of knowledge, preparedness, and practice. We used survey and focus groups responses to understand participant perceptions of ECHO effectiveness.

Data Analysis. We analyzed survey data using summary statistics. We compared pre- and post-ECHO data among participants who attended at least $50 \%$ of sessions and completed baseline and follow-up surveys. We examined the distribution of the ordinal outcomes and dichotomized outcomes based on median values, using baseline frequencies to determine cut-point for pre-post items (Table 3). We used paired $t$ tests and tests of proportions for continuous and dichotomous variables, respectively, in STATA. ${ }^{23}$ For missing data, we imputed the lowest scale code/ item (e.g., never). We transferred short-answer survey responses and focus group transcripts from all participants, regardless of attendance, to Atlas.ti. ${ }^{24}$ The study team first reviewed survey responses and generated preliminary codes. Three coders (AP, RL, MM) independently coded transcripts and met in dyads to reconcile codes. We then applied codes to focus group transcripts, using the process to confirm or elaborate short-answer findings. Finally, we performed a thematic analysis of the entire data set, using an inductive approach at the semantic level. If participants joined multiple cohorts, we included their survey data multiple times. southwest Washington hospital, comprised of 22 urban, 8 rural, and 3 frontier hospitals (Table 2).

One hundred eight of 143 registrants completed the baseline survey. At baseline, 42 (38.9\%) participants reported that patients with opioid use disorder (OUD) admitted to their hospital were offered to start buprenorphine-naloxone at least some of the time, and 42 (38.9\%) reported that patients were offered to start methadone at least some of the time. Of the 56 registered clinicians (MD, DO, NP, PA), 30 (53.6\%) had a buprenorphine waiver.

\section{Feasibility}

One hundred and twenty-seven (88.8\%) registrants attended at least one ECHO session, 96 (67.1\%) attended at least half, and $74(51.7 \%)$ attended at least half of the sessions and completed baseline and follow-up surveys. Many participants invited colleagues to join current or later cohorts.

\section{Acceptability}

Participants were highly satisfied with ECHO. Mean persession satisfaction was 4.3 [range 1 (poor) to 5 (excellent)]. Among the 94 participants who completed post-ECHO evaluations, 88 (94\%) said they were likely to recommend the SUD in hospital care ECHO to colleagues. Qualitative data provided important context for those scores. Participants valued training on topics on which they had little training, including withdrawal management, medication for opioid use disorder (MOUD) methadone and buprenorphine, harm reduction, and traumainformed care. Participants also valued point-of-care resources

Table 2 Participant Characteristics

\begin{tabular}{lll}
\hline \hline Characteristics & $\begin{array}{l}\text { Registered } \\
(\boldsymbol{n}=\mathbf{1 4 3})\end{array}$ & $\begin{array}{l}\text { Attended } \geq \mathbf{5 0 \%} \text { of } \\
\text { sessions }(\boldsymbol{n}=\mathbf{9 6})\end{array}$ \\
\hline Mean age, years (range) & $44.7(25-77)$ & $44.8(25-77)$ \\
Female gender, $n(\%)$ & $104(72.7)$ & $69(71.9)$ \\
Non-Hispanic white, $n(\%)$ & $102(71.3)$ & $66(68.8)$ \\
Discipline, $n(\%)$ & $56(39.2)$ & $43(44.8)$ \\
MD/DO/NP/PA & $29(20.3)$ & $20(20.8)$ \\
Social worker/counselor & $19(13.3)$ & $9(9.4)$ \\
Nurse & $13(9.1)$ & $4(4.2)$ \\
Hospital leadership & $13(9.1)$ & $10(10.4)$ \\
Pharmacist & $4(2.8)$ & $2(2.1)$ \\
Peer/outreach coordinator & $5(3.4)$ & $8(8.3)$ \\
Quality improvement staff & $1(0.7)$ & $0(0.0)$ \\
Physical therapist & & \\
Work role, $n(\%)$ & $30(21.0)$ & $20(20.8)$ \\
Executive staff & $30(21.0)$ & $17(17.7)$ \\
Manager/supervisor & $111(77.6)$ & $77(80.2)$ \\
Provide direct patient care & & \\
Hospital region, $n$ (\%) & $92(64.3)$ & $57(59.4)$ \\
Portland metro & $46(32.2)$ & $35(36.5)$ \\
Non-Portland area, Oregon & $5(3.5)$ & $3(3.1)$ \\
Southwest Washington & & \\
\hline
\end{tabular}

\section{RESULTS}

We recruited 143 registrants to three cohorts between January and September 2019. Seven individuals participated twice.Participants drew from 32 of Oregon's 62 hospitals and one 
and practical tools (e.g., methadone policies, tools for buprenorphine induction), noting that they were working to adopt and implement these tools in their hospitals.

Many participants highlighted the value of interprofessional ECHO faculty who modeled team-based care and attention to the use of nonstigmatizing language. As one hospital administrator-participant said:

Everyone providing the ECHO took every opportunity to invite people into their compassion... [ECHO is] a master class in how to speak with folks who are curious but uncertain. I really appreciate the modeling.

Notably, we modified the curriculum to respond to participant feedback, which likely further increased acceptability. Partway through cohort 1 , some participants shared that the skills we were teaching still felt abstract, so we added a faculty role play to model SUD assessments and patient-provider discussions about MOUD. Many participants found the role play "incredibly valuable," though some felt the patient was "too easy." Thus, in cohorts 2 and 3 , we added a second role play depicting common conflicts with an angry patient. ${ }^{18}$ Other modifications included extending cohorts 2 and 3 to 12 weeks (from 10) to allow additional topics including methamphetamine use disorder and a second role play.

\section{Effects on Knowledge and Practice}

At baseline, most participants reported low preparedness to diagnose, assess, and treat SUD and low MOUD prescribing (Table 3). Post-ECHO, participants felt more prepared to treat SUD, and knowledge scores increased. Prescribing, however, did not change post-ECHO. Qualitative evaluation identified three main themes related to knowledge and skills, specifically: (1) use of MOUD in hospital care, (2) importance of patient-centered care for people who use drugs, and (3) harm reduction.

How to Use MOUD in Hospital Care. Throughout, ECHO emphasized that all patients with opioid use disorder should be offered MOUD initiation and linkage to treatment after discharge. Participants felt ECHO increased their understanding of how to dose and initiate methadone and buprenorphine. As one physician-participant described:

One of the things that [ECHO] really did for me was fill in a lot of knowledge gaps, particularly around using methadone, which I never have done before... and giving us practical steps to begin doing that in the hospital.

ECHO also clarified common misunderstandings of federal rules surrounding the use of methadone and buprenorphine inpatient and after discharge. Some participants had not previously understood that it is legal to prescribe buprenorphine
Table 3 ECHO Effectiveness

\begin{tabular}{|c|c|c|c|}
\hline \multirow[t]{2}{*}{ Pre-/post-ECHO ${ }^{*}$} & \multirow{2}{*}{$\begin{array}{l}\text { Pre } \\
\text { Mean } \\
\text { (SD) }\end{array}$} & \multirow{2}{*}{$\begin{array}{l}\text { Post } \\
\text { Mean } \\
\text { (SD) }\end{array}$} & \multirow{2}{*}{$\begin{array}{l}p \\
\text { value }\end{array}$} \\
\hline & & & \\
\hline Knowledge; $\%$ correct $(n=74)$ & $\begin{array}{l}76 \% \\
(21 \%)\end{array}$ & $\begin{array}{l}82 \% \\
(18 \%)\end{array}$ & 0.002 \\
\hline Very prepared to: $(n=62)^{\dagger}$ & $\begin{array}{l}\text { Pre } \\
n(\%)\end{array}$ & $\begin{array}{l}\text { Post } \\
n(\%)\end{array}$ & $\begin{array}{l}p \\
\text { value }\end{array}$ \\
\hline Make a diagnosis of SUD & $25(40)$ & $35(56)$ & 0.072 \\
\hline $\begin{array}{l}\text { Discuss community treatment } \\
\text { options }\end{array}$ & $16(26)$ & $28(45)$ & 0.024 \\
\hline Discuss overdose prevention & $14(23)$ & $33(53)$ & $>001$ \\
\hline Discuss harm reduction & $8(13)$ & $33(53)$ & $\begin{array}{l}> \\
0.001\end{array}$ \\
\hline \multicolumn{4}{|l|}{$\begin{array}{l}\text { MD, DO, NP, and PA prescribing } \\
\text { practice, frequency }(n=39)\end{array}$} \\
\hline $\begin{array}{l}\text { Initiate methadone at least } \\
\text { sometimes }\end{array}$ & $8(20)$ & $10(26)$ & 0.59 \\
\hline $\begin{array}{l}\text { Initiate buprenorphine at least } \\
\text { sometimes }\end{array}$ & $14(36)$ & $20(51)$ & 0.17 \\
\hline $\begin{array}{l}\text { Always offer naloxone to those at } \\
\text { risk of opioid overdose }{ }^{\S}\end{array}$ & $11(28)$ & $14(36)$ & 0.46 \\
\hline Always refer to OUD treatment ${ }^{\S}$ & $3(8)$ & $5(13)$ & 0.45 \\
\hline \multicolumn{2}{|l|}{ Post-ECHO $(n=82)^{\|}$} & $\begin{array}{l}\text { Post } \\
n(\%)\end{array}$ & \\
\hline $\begin{array}{l}\text { ECHO participation helped me } \\
\text { understand resources to treat SUD } \\
\text { in my community a lot }\end{array}$ & - & $45(55)$ & - \\
\hline $\begin{array}{l}\text { ECHO participation changed the } \\
\text { way I think about caring } \\
\text { for people with SUD }\end{array}$ & - & $59(72)$ & - \\
\hline $\begin{array}{l}\text { ECHO participation changed my } \\
\text { personal practice }\end{array}$ & - & $58(71)$ & - \\
\hline $\begin{array}{l}\text { ECHO participation effected the } \\
\text { way that providers in my } \\
\text { hospital work together to care for } \\
\text { patients with SUD a lot }\end{array}$ & - & $29(35)$ & - \\
\hline
\end{tabular}

$S D=$ standard deviation; $O U D=$ opioid use disorder; $S U D=$ substance use disorder

*Includes participants who attended 50\% or more of sessions and completed baseline and follow-up surveys

†Very prepared $v$ s. less than very prepared (very unprepared, somewhat unprepared, neither, somewhat prepared) dichotomized from a 5-point Likert scale

At least sometimes (sometimes, often, always) vs. less than sometimes (rarely, never) dichotomized from a 5-point Likert scale

$\$$ Always vs. less than always (never, rarely, sometimes, often) dichotomized from a 5-point Likert scale

"Includes participants who attended 50\% or more of sessions and completed the follow-up survey

${ }^{\top}$ A lot vs. less than a lot (not at all, a little, some) dichotomized from 4point Likert items

${ }^{\#}$ Yes vs. no

and methadone during hospitalization. As one pharmacistparticipant described, "It came as news to me as a pharmacist that we could prescribe methadone in hospital to treat withdrawal." ECHO also clarified prescribing practices at time of hospital discharge, clarifying - and reiterating through case discussions - that it is not legal to prescribe methadone for OUD at discharge (and instead must be administered through an opioid treatment program) and that current regulations require providers to have a federal waiver to prescribe buprenorphine in the setting of OUD at the time of discharge.

Even among participants who prescribed MOUD before ECHO, many lacked knowledge or confidence to titrate medications to effective doses and were unsure how to manage 
acute pain in the setting of opioid use disorder. ECHO improved this. As one physician-participant described, because of ECHO:

I feel comfortable adding acute pain meds on top of chronic OUD meds- and splitting OUD meds [multiple times per day] for better pain control- and being OK with rapid taper of acute meds knowing chronic meds will prevent withdrawal.

Others described sharing new knowledge from $\mathrm{ECHO}$ with coworkers. Another physician-participant described educating a colleague about the benefits of full opioid agonists for acute pain management in patients on buprenorphine-naloxone:

I had a patient a couple weeks ago that had a horrific arm wound and she had been discharged without any pain medicine. I pulled the surgeon out of the room and asked why not. She sort of lowered her voice and she said 'I can't give pain medicine with suboxone.' It was really great to be able to have that conversation.

For some participants, $\mathrm{ECHO}$ was "a really useful launching point" and "made the difference between treating and not treating OUD in the hospital." As one nurse practitioner-participant described, "I couldn't even attempt what I'm doing without this education and insight from experienced practitioners."

Others felt that they needed more to change practice. As one physician-participant described: "Yes we want to do it [prescribe buprenorphine-naloxone], but we are scared. We don't want to screw up, and we feel like we need more support to be able to actually provide care."

Patient-Centered Care for People Who Use Drugs. Training allowed participants to understand patients with SUD as humans who are struggling, and supported them with difficult patient interactions. Many felt that ECHO, and particularly the peer voice, helped them contextualize and empathize with patients' substance use and their behaviors. As one physicianparticipant noted, I now "approach every patient as if they have had a past traumatic experience and am sensitive to the impact that that can have on their hospital experience." Participants reflected that ECHO shed new light on their own preconceived biases and judgment toward people who use drugs, which "kind of melted away" as they reframed challenging patient behaviors as a response to trauma and stress of hospitalization. As a pharmacist-participant described:

[ECHO] opened my eyes to things we weren't doing correctly in the hospital.... [Before], it was super easy to objectify the person and say they are causing their own problem and there's nothing we can do. Because we feel helpless, we don't know what else to do.
Many participants reflected on the value of training around trauma-informed care. As one physicianparticipant noted:

I loved being told by the presenters, 'it's okay to stop the interaction and leave the room when it's not going well.' I needed to hear that because I try too hard to make it go from negative to positive when I have an activated, angry patient. I will now try a cool off time and come back.

Understanding Harm Reduction as a Valid Approach to Treatment. For many, ECHO introduced the idea of harm reduction or expanded it beyond simply syringe exchange. In cases, participants commonly grappled with the inherent tensions between hospital culture, zero-tolerance substance use policies, and harm reduction. Harm reduction concepts, coupled with framing addiction as a treatable chronic disease, allowed many participants to move past an abstinence-only approach where it was their "job to stop patients from using drugs." This shift allowed some to de-escalate crises and feel more like a caregiver, "not a cop." As one nurse-participant described:

Many times we struggle with how to treat patients that are using [drugs] in the hospital. It's a complex issue... [and] it can be difficult to know how far to go to stop this from happening and whether it's really our job to stop patients from using. This can create a great deal of stress on the staff. To hear OHSU's nursing care plan puts less importance on this and more focus with communicating with the patient on what they feel their boundaries should be gives me a better plan for these patients.

Participants appreciated practical teaching, including specifics of suggesting some patients smoke instead of inject substances or access water from the tank instead of the front of the toilet bowl. As one social worker summarized: "[I learned] it's OK to consider harm reduction as a valid treatment goal."

\section{Lessons Learned and Implications for Spread}

In addition to identifying the knowledge and skills the participants gained, qualitative work also revealed additional ECHO advantages and limitations. Specifically, qualitative work identified ECHO as (1) an important resource for decreasing provider isolation while (2) also clarifying ECHO limitations regarding changing hospital policies, leadership and provider culture, and SUD resources.

Provider Isolation and Building Community of Practice. Many participants emphasized that ECHO bestowed a sense of hopefulness and growth. Many participants described being the first in their hospitals to "think of OUD as something we actively 
manage in the hospital." This could be difficult and ECHO was a support. As one physician-participant summarized, "It was helpful to know there are many people around the state dealing with the same issues." Another physician-participant described:

Hearing other people's stories... reinforced that I'm not alone in not feeling confident or not treating patients correctly, that we're all way behind... I've still got a lot to learn, but I've got a lot more confidence and I'm hopeful to grow our program.

\section{Role for Supportive Hospital Leadership, Policies, and SUD}

Resources. While participants identified ECHO as an important resource, they also recognized that hospital leadership, policies, and culture could impede SUD care delivery, even as providers expanded their knowledge and skills. Many participants felt that reform would require "philosophical change," noting that most staff continue to "err on the side of punitive and suspicious with our SUD patients." As one nurse-participant described:

There is still a big community of medical providers who function from a place of 'it's their choice, their consequences.' It is exhausting.

Hospital policies were a key factor in participants' ability to implement evidence-based addictions care. Many participants struggled with MOUD, smoking, and visitor policies. A few participants changed policies during the 12-week ECHO. As one physician-participant described, because of ECHO:

...we [implemented] a methadone policy for the hospital (looks a lot like OHSU's)... as a result people have been able to prescribe in the hospital.... I had never used methadone for [opioid use disorder] prior to that.

Others, however, described persistent policy barriers. As one physician-participant described:

I feel like I am stuck way back in the dark ages... It still isn't clear if we are even allowed to prescribe methadone to patients that are not already on it. Apparently the residents have been told by a number of pharmacists that they are not allowed to start methadone for OUD. We are not really sure. The policy is from 2002.

Many also highlighted a need for senior leadership to invest resources. As one nurse practitioner-participant described: "I don't know how to get through to leadership. We are a regional medical center and there are only 2 physicians with expertise in these medications."

Lack of protected time and resources to develop and lead hospital-based SUD efforts was another common barrier. Some participants, particularly in rural areas, described treatment gaps across the communities at-large as severely limiting. Many rural hospitals had no buprenorphinewaivered providers and lacked adequate post-hospital referral pathways. As one rural physician-participant described: "we can get folks started but we have nowhere to send them afterwards."

\section{DISCUSSION}

We developed and implemented an SUD in hospital care ECHO led by interprofessional faculty that included diverse participants across urban, rural, and frontier hospitals. ECHO was highly rated with robust attendance. Participants identified substantial gains in knowledge and skills to treat SUDs, particularly regarding the use of MOUD, patient-centered communication, and understanding of harm reduction as an important treatment intervention. Participants gained new insights about their own views of SUD and especially felt that peer faculty perspectives deepened their empathy and enriched their understanding of pre-existing biases. We also found that for many participants, absent hospital leadership buy-in and policy change, gains in knowledge were insufficient to substantially alter practice during the 12-week ECHO.

Earlier work describes experiences implementing hospitalbased addictions care at single-site, urban, academic medical centers. ${ }^{10,25,26}$ To our knowledge, this is the first study to evaluate a statewide effort to implement hospital-based addictions care or to describe implementation experiences across community, rural, and frontier hospitals. Earlier studies describe using ECHO to educate primary care providers about $\mathrm{SUD}^{27-30}$; ours is the first description of ECHO to support SUD care among hospital providers. Like ambulatory ECHO experiences, we identified challenges of negative staff attitudes toward SUD ${ }^{27}$ lack of leadership support, ${ }^{27}$ and limited rural treatment resources. Our experience also exposed the ways in which confusion around hospital policies and federal regulations surrounding methadone and buprenorphine can impede hospital care. Interestingly, policy, culture, and leadership barriers identified by community hospital participants in our ECHO mirror some barriers described at academic medical centers. ${ }^{31}$ Both identified negative staff attitudes toward people who use drugs, restrictive and misinterpreted methadone and buprenorphine policies, referral pathways to treatment after discharge, and sufficient financing and resources as key issues. ${ }^{31}$ Rural and small community hospitals, however, have fewer resources. Our study also builds on literature describing widespread negative provider attitudes toward people with substance use, ${ }^{32}$ and supports that ECHO - especially peer faculty - can shift that. Finally, this study builds on growing literature about training healthcare providers - and especially physicians ${ }^{33-35}$ - in addictions care. It is novel in that it targets interprofessional hospital providers, uses case-based learning with cases identified in real time by providers newly engaging in the treatment of 
SUDs, and focuses on continuing education for practicing professionals.

Our study has several important limitations. It is a pre-post study with no comparison group, so it is possible that knowledge, preparedness, and practice changes were due to other causes. However, our qualitative evaluation asked specifically about participants' experiences, lessons, and practice changes resulting from ECHO. Second, we describe self-reported provider-level outcomes, which may be subject to bias, and we do not measure patient-level outcomes. Future work should explore objective provider-level outcomes and patient experiences associated with provider participation in ECHO. Third, our evaluation was not designed to fully explore the characteristics of providers and hospitals that make ECHO most effective. Future work to understand which interventions in which settings are most effective is critical to long-term adoption of SUD care across all hospitals. Finally, our study lacks longterm follow-up. It is possible that the ECHO effect wanes over time. It is also possible that a 12 -week ECHO is not enough time to detect changes in hospital policies or for providers to complete buprenorphine waiver trainings or initiate SUD consultation services. Future work should evaluate ECHO effectiveness. Notably, in informal follow-up after ECHO, many participants have obtained a buprenorphine waiver, and a few have secured resources for addiction consult services.

Our study has implications for hospital providers, hospital leaders, and policy makers, especially as regional health systems and states push to broadly adopt hospital-based SUD care. ECHO can be a useful tool to increase SUD knowledge and preparedness and can be a starting point for providers looking to implement hospital-based addictions care. That many participants found much-needed energy and support from a statewide, interprofessional community of practice within ECHO highlights the potential for ECHO to support clinician-leaders to drive change.

Our findings suggest that hospital leaders' decisions, actions, and attention are critical to supporting evidencebased, nondiscriminatory care for people with SUD. That so many ECHO participants struggled against outdated, nonevidence-based policies underscores the need for hospital leaders to prioritize the review of MOUD policies and assure that they adhere to national treatment guidelines. ${ }^{36}$ All participants identified individual and structural stigma toward people using drugs as key challenges. Hospital leaders can address stigma and support culture change by normalizing addiction treatment as part of usual hospital care,${ }^{10}$ promoting the use of nonstigmatizing language, ${ }^{37}$ and supporting clinical champions invested in addressing individual and population health needs of people with SUD. Furthermore, hospital leaders are uniquely positioned to address limited treatment pathways by engaging community partners to develop and sustain hospitalcommunity partnerships that address SUD needs across the care continuum.

Finally, our study reflects the widespread need for SUDfocused workforce development across disciplines. Baseline preparedness to treat SUD was low, even among motivated providers willing to participate in a 12-week ECHO, over half of whom already had a buprenorphine waiver. Furthermore, concepts of trauma-informed care and harm reduction were new for many participants. The training gaps evidence by our findings underscore the imperative to treat addiction in hospitals - both to address treatment gaps and because hospitals are crucial training environments for physicians, nurses, and other healthcare professionals. ${ }^{38}$

Our findings support ECHO as a feasible and acceptable way to build capacity across geographically diverse, interprofessional hospital providers, and suggest a role for local, state, and national hospital organizations to fund and expand ECHO for this purpose. Our study also suggests that hospital teams may need more than training to support broad-scale implementation of addictions care. Additional strategies might include in-person or telehealth consultation from addiction specialists who could see complex patients and provide real-time clinical support, practice facilitation from experienced hospital-based addictions teams, and dedicated funding for local SUD champions. Understanding what additional strategies can support broad-scale adoption of SUD care across community urban, rural, and frontier hospitals is an area ripe for future research.

Acknowledgments: The authors would like to acknowledge the ECHO participants; ECHO faculty, lecturers, and fellows including Jackie Sharpe, Stacey Mahoney, Susannah Lujan-Bear, Sean Mahoney, Brittney Caldera, Traci Lundy, Lisa Whitmore, Juliana Wallace, Emily Skogrand, Dr. Ximena Levander, Dr. Jennifer Hartley, Dr. Raymundo Garcia-Dwyer, Dr. Casey Ferguson, Dr. Jonathan Robbins, Dr. Alyson Smith, and Dr. Cydney Heims; and Marie-Annick Yagapen and Dr. Gabriel Plourde for their support. They would also like to thank Maggie McClain McDonnell, Terry Wilson, Miriam Wolf, and the entire Oregon ECHO Network; Katie Harris and Danielle Meyers from the Oregon Association for Hospitals and Health Systems (OAHHS); Caroline King MPH for her help with statistical analysis and review; Dr. Jennifer McNeely for help developing surveys; Dr. Christina Nicolaidisfor her help with evaluation; and, Pamela Pierce for her assistance building a resource repository. Finally, the authors would like to thank Dr. P. Todd Korthuis for his support in obtaining funding for this ECHO.

Corresponding Author: Honora Englander, MD; Division of Hospital Medicine, Department of Medicine, Oregon Health \& Science University, Portland, OR, USA (e-mail: englandh@ohsu.edu).

Funding $\mathrm{ECHO}$ was funded through Oregon SAMHSA/CSAT State Targeted Response contract (1H79T1080258). The project described was supported by the National Center for Advancing Translational Sciences (NCATS), National Institutes of Health, through Grant Award Number UL1TRO02369. The content is solely the responsibility of the authors and does not necessarily represent the official views of the NIH.

\section{Compliance with Ethical Standards:}

Conflict of Interest: The authors declare that they do not have a conflict of interest. 


\section{REFERENCES}

1. Owens PL, Fingar KR, McDermott KW, Muhuri PK, Heslin KC. Inpatient stays involving mental and substance use disorders, 2016. Statistical brief \#249. Healthcare Cost and Utilization Project. 2016. https://www.hcup-us.ahrq.gov/reports/statbriefs/ sb249-Mental-Substance-Use-Disorder-Hospital-Stays-2016.pdf. Accessed 21 May 2019.

2. Larochelle MR, Bernstein R, Bernson D, Land T, Stopka TJ, Rose AJ, et al. Touchpoints - opportunities to predict and prevent opioid overdose: a cohort study. Drug Alcohol Depend. 2019;204:107537. https://doi. org/10.1016/j.drugalcdep.2019.06.039

3. Englander H, Weimer M, Solotaroff R, Nicolaidis C, Chan B, Velez C, et al. Planning and designing the Improving Addiction Care Team (IMPACT) for hospitalized adults with substance use disorder. J Hosp Med. 2017;12(5):339-42https://doi.org/10.12788/jhm.2736

4. Velez CM, Nicolaidis $\mathbf{C}$, Korthuis PT, Englander $\mathbf{H}$. "It's been an experience, a life learning experience": a qualitative study of hospitalized patients with substance use disorders. J Gen Intern Med. 2017;32(3):296-303. https://doi.org/10.1007/s11606-016-3919-4

5. Collins D, Alla J, Nicolaidis C, Gregg J, Gullickson DJ, Patten AM, et al. "If it wasn't for him, I wouldn't have talked to them": qualitative study of addiction peer mentorship in the hospital. J Gen Intern Med. 2019. https://doi.org/10.1007/s11606-019-05311-0

6. Hyshka E, Morris H, Anderson-Baron J, Nixon L, Dong $\mathbf{K}$, Salvalaggio G. Patient perspectives on a harm reduction-oriented addiction medicine consultation team implemented in a large acute care hospital. Drug Alcohol Depend. 2019;204:107523. https://doi.org/10.1016/j.drugalcdep.2019.06.025

7. Wakeman SE, Metlay JP, Chang Y, Herman GE, Rigotti NA. Inpatient addiction consultation for hospitalized patients increases post-discharge abstinence and reduces addiction severity. J Gen Intern Med. 2017;32(8):909-16. https://doi.org/10.1007/s11606-017-4077-Z

8. Englander H, Dobbertin K, Lind BK, Nicolaidis C, Graven P, Dorfman C, et al. Inpatient addiction medicine consultation and post-hospital substance use disorder treatment engagement: a propensity-matched analysis. J Gen Intern Med. 2019;34(12):2796-803. https://doi.org/10. 1007/s11606-019-05251-9

9. King C, Nicolaidis C, Korthuis PT, Priest KC, Englander H. Patterns of substance use before and after hospitalization among patients seen by an inpatient addiction consult service: A latent transition analysis. J Subst Abuse Treat. 2020;118:108121. https://doi.org/10.1016/j.jsat.2020. 108121

10. Englander H, Collins D, Perry SP, Rabinowitz M, Phoutrides E, Nicolaidis C. "We've learned it's a medical illness, not a moral choice": qualitative study of the effects of a multicomponent addiction intervention on hospital providers' attitudes and experiences. J Hosp Med. 2018;13(11):752-8. https://doi.org/10.12788/jhm.2993

11. Rapoport AB, Rowley CF. Stretching the scope - becoming frontline addiction-medicine providers. N Engl J Med. 2017;377(8):705-7. https:// doi.org/10.1056/NEJMp1706492

12. Botticelli M, Bottlieb M, Laderman M. Effective strategies for hospitals responding to the opioid crisis. Institute for Healthcare Improvement and The Grayken Center for Addiction at Boston Medical Center, Boston; 2019. www.ihi.org. Accessed 12 April 2020.

13. Priest KC, McCarty D. Role of the hospital in the 21st century opioid overdose epidemic: the addiction medicine consult service. J Addict Med. 2019;13(2):104-12. https://doi.org/10.1097/adm.0000000000000496

14. Komaromy M, Ceballos V, Zurawski A, Bodenheimer T, Thom DH, Arora S. Extension for Community Healthcare Outcomes (ECHO): a new model for community health worker training and support. J Public Health Policy. 2018;39(2):203-16. https://doi.org/10.1057/s41271-017-0114-8

15. Oregon ECHO Network [Internet]. 2018. www.oregonechonetwork.org. Accessed 13 April 2020.

16. Englander $\mathbf{H}$, Mahoney $\mathbf{S}$, Brandt $\mathbf{K}$, Brown $\mathbf{J}$, Dorfman $\mathbf{C}$, Nydahl A, et al. Tools to support hospital-based addiction care: core components, values, and activities of the Improving Addiction Care Team. J Addict Med. 2019;13(2):85-9. https://doi.org/10. $1097 /$ adm.0000000000000487

17. Project ECHO: A Revolution in Medical Education and Care Delivery [Internet]. The University of New Mexico; 2019. https://echo.unm.edu/ Accessed 15 April 2020

18. Oregon Health \& Science University. Digital Collections. https://scholararchive.ohsu.edu/collections/2v23vv13z?locale=en. Accessed 23 July 2020.

19. Substance Abuse and Mental Health Services Administration. TI-17-014 State Targeted Response to the Opioid Crisis Grants (Opioid STR)
Individual Grant Awards; 2017. https://www.samhsa.gov/sites/default/files/grants/pdf/other/ti-17-014-opioid-str-abstracts.pdf. Accessed 1 July 2020.

20. Hanson WE, Creswell JW, Clark VLP, Petska KS, Creswell JD. Mixed methods research designs in counseling psychology. J Couns Psychol 2005;52(2):224-35. https://doi.org/10.1037/0022-0167.52.2.224

21. Wakeman SE, Pham-Kanter G, Donelan K. Attitudes, practices, and preparedness to care for patients with substance use disorder: results from a survey of general internists. Subst Abus. 2016;37(4):635-41. https://doi.org/10.1080/08897077.2016.1187240

22. Continuing Education Available at OHSU: Continuing Medical Education. Oregon Health \& Science University. https://www.ohsu.edu/school-of-medicine/cpd/continuing-education-available-ohsu. Accessed 24 June 2020.

23. StataCorp. 2017. Stata Statistical Software: Release 15. College Station TSL.

24. ATLAS.ti 8 Windows. 2017. Scientific Software Development GmbH.

25. Noska A, Mohan A, Wakeman S, Rich J, Boutwell A. Managing opioid use disorder during and after acute hospitalization: a case-based review clarifying methadone regulation for acute care settings. J Addict Behav Ther Rehabil. 2015;4(2). https://doi.org/10.4172/2324-9005.1000138

26. Wakeman SE, Kanter GP, Donelan K. Institutional substance use disorder intervention improves general internist preparedness, attitudes, and clinical practice. J Addict Med. 2017;11(4):308-14. https://doi.org/ 10.1097/adm.0000000000000314

27. Shea CM, Gertner AK, Green SL. Barriers and perceived usefulness of an ECHO intervention for office-based buprenorphine treatment for opioid use disorder in North Carolina: a qualitative study. Subst Abus. 2019:1-11. https://doi.org/10.1080/08897077.2019.1694617

28. Komaromy M, Duhigg D, Metcalf A, Carlson C, Kalishman S, Hayes L et al. Project ECHO (Extension for Community Healthcare Outcomes): a new model for educating primary care providers about treatment of substance use disorders. Subst Abus. 2016;37(1):20-4. https://doi.org/ 10.1080/08897077.2015.1129388.

29. Covell N, Foster F, Margolies P, Lopez L, Dixon L. Using distance technologies to facilitate a learning collaborative to implement stagewise treatment. Psychiatr Serv. 2015;66(6):645-8. https://doi.org/10.1176/ appi.ps.201400155

30. Chaple MJ, Freese TE, Rutkowski BA, Krom L, Kurtz AS, Peck JA et al. Using ECHO Clinics to Promote Capacity Building in Clinical Supervision. Am J Prev Med. 2018;54(6 Suppl 3):S275-s80. https://doi. org/10.1016/j.amepre.2018.01.015

31. Priest KC, Englander H, McCarty D. "Now hospital leaders are paying attention": a qualitative study of internal and external factors influencing addiction consult services. J Subst Abus Treat. 2020;110:59-65. https:// doi.org/10.1016/j.jsat.2019.12.003

32. van Boekel LC, Brouwers EP, van Weeghel J, Garretsen HF. Stigma among health professionals towards patients with substance use disorders and its consequences for healthcare delivery: systematic review. Drug Alcohol Depend. 2013;131(1-2):23-35. https://doi.org/10.1016/j. drugalcdep.2013.02.018

33. Polydorou S, Gunderson EW, Levin FR. Training physicians to treat substance use disorders. Curr Psychiatry Rep. 2008;10(5):399-404. https://doi.org/10.1007/s11920-008-0064-8

34. Gorfinkel L, Klimas J, Reel B, Dong H, Ahamad K, Fairgrieve C, et al. In-hospital training in addiction medicine: a mixed-methods study of health care provider benefits and differences. Subst Abus. 2019:1-7. https://doi.org/10.1080/08897077.2018.1561596

35. Klimas J, Small W, Ahamad $\mathbf{K}$, Cullen W, Mead A, Rieb L, et al. Barriers and facilitators to implementing addiction medicine fellowships: a qualitative study with fellows, medical students, residents and preceptors. Addict Sci Clin Pract. 2017;12(1):21. https://doi.org/10. 1186/s13722-017-0086-9

36. Substance Abuse and Mental Health Services Administration. Tip 63: Medications for Opioid Use Disorder - Executive Summary. https://store. samhsa.gov/system/files/sma18-5063exsumm.pdf. Accessed 20 March 2020

37. Changing the Narrative [Internet]. Health in Justice Action Lab: Changing the Narrative Initiative, Northeastern University School of Law; 2019. www.changingthenarrative.news/. Accessed 15 April 2020.

38. Data Resource Book Academic Year 2018-2019. Accredication Council for Graduate Medical Education (ACGME), Chigaco, Illinois; 2019. https:// www.acgme.org/About-Us/Publications-and-Resources/Graduate-Medical-Education-Data-Resource-Book/GraduateMedicalEducation/GraduateMedicalEducationDataResourceBook. Accessed 17 April 2020.

Publisher's Note: Springer Nature remains neutral with regard to jurisdictional claims in published maps and institutional affiliations. 